\title{
Éditorial : Rio+20, dépasser l'horizon de la conférence
}

Helga-Jane Scarwell

\section{Q OpenEdition}

10 Journals

\section{Édition électronique}

URL : http://journals.openedition.org/developpementdurable/9341

DOI : 10.4000/developpementdurable.9341

ISSN : 1772-9971

Éditeur

Association DD\&T

\section{Référence électronique}

Helga-Jane Scarwell, «Éditorial : Rio+20, dépasser l'horizon de la conférence », Développement durable et territoires [En ligne], Vol. 3, n² 2 I Juillet 2012, mis en ligne le 10 juillet 2012, consulté le 22 septembre 2020. URL : http://journals.openedition.org/developpementdurable/9341 ; DOI : https://doi.org/ 10.4000/developpementdurable.9341

\section{Ce document a été généré automatiquement le 22 septembre 2020}

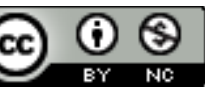

Développement Durable et Territoires est mis à disposition selon les termes de la licence Creative Commons Attribution - Pas d'Utilisation Commerciale 4.0 International. 


\title{
Éditorial : Rio+20, dépasser l'horizon de la conférence
}

\author{
Helga-Jane Scarwell
}

1 Quarante ans après la conférence de Stockholm (1972), 20 ans après Rio (1992) et «Notre avenir à tous» (1987), la quatrième Conférence des Nations Unies sur le développement durable (CNUDD dite aussi Rio+20) intitulée «le futur que nous voulons ", s'est tenue des 20 au 22 juin 2012 à Rio de Janeiro. Elle constituait assurément un événement majeur sur la scène internationale car chacun s'accorde à dire qu'il est urgent d'agir ou de réagir pour alerter sur l'état inquiétant de la planète. La communauté internationale convoquée au chevet de la planète ne devait-elle pas s'efforcer de définir un nouvel agenda pour le développement soutenable? Aussi cruciaux que soient les problèmes et enjeux agrégés par la problématique du développement durable, Rio+20 ne devait-il pas être aussi un espace de réflexion, de discussion et d'expérimentation qui dépasse l'urgence, le catastrophisme écologique et, plus globalement, l'horizon de la Conférence et le bouillonnement lié à ce type de manifestation pour poser la question du modèle de règlement intergouvernemental des problèmes soulevés?

2 Si l'on s'en tient strictement au bilan de ce sommet, il y a plusieurs façons d'en juger les résultats.

On pourrait par exemple les appréhender en soulignant que Rio 2012 était précédé d'un Sommet des peuples « pour la justice sociale et environnementale, contre la marchandisation de la vie et pour la défense des biens communs", constituant ainsi une opportunité de réfléchir aux outils propres à démanteler les politiques qui perpétuent les inégalités entre les pays, et à ceux qui favoriseraient la progression de l'égalité et l'élargissement des opportunités pour les individus partout dans le monde dans un cadre de référence en commun. On pourrait encore rappeler que le sommet s'est déroulé dans un contexte de crises profondes, aux dimensions multiples (politique, économique, sociale, etc.) susceptibles de reléguer au second plan les préoccupations écologiques des principaux dirigeants internationaux. D'autant que la question de l'interconnexion ou de la 
relation entre les sphères économiques, monétaires et écologiques revêt une importance stratégique. Cependant, nous ne la développerons pas dans cet éditorial.

4 Nous nous attarderons davantage sur les deux axes principaux du programme qui portaient sur l'économie verte et le renforcement des cadres institutionnels du développement durable.

\section{L'économie verte}

5 Le thème de l'économie verte ${ }^{1}$ pourrait-être interprété comme le signe de la prise de conscience de l'urgence écologique à trouver des alternatives à l'économie «brune » et de la volonté d'y répondre par des solutions concrètes. Il pourrait tout aussi bien apparaître comme une fuite technologique. Depuis quelques années, l'économie verte a le vent en poupe, pourtant elle souffre d'imprécisions conceptuelles (Aubertin, 2012) que son absence de définition dans le Draft Zero, qui préfigure la déclaration finale du sommet de Rio, n'a non seulement pas démenti mais a suscité de nombreuses contestations parmi les pays du Sud rassemblés au sein du G 77 .

6 On pourrait, de facto, s'interroger sur ce que les gouvernements comprennent par économie verte? Serait-ce un nouveau départ en matière de développement durable sous le label " économie verte ", autrement dit une opportunité de croissance " verte ", ou s'agit-il d'une rhétorique internationale masquant la poursuite de politique et d'économie non durable sous le label « économie verte » ou encore une nouvelle étape dans la marchandisation et la financiarisation de la vie et du vivant, la marchandisation de la nature (Gleizes, 2012) ? Manifestement, cette "économie verte» est déjà à l'œuvre (marchés carbone, agro-carburants) et telle qu'elle est posée dans les documents officiels, elle a tendance à considérer la nature comme un capital à gérer de la manière la plus efficiente.

\section{Le renforcement des cadres institutionnels du développement durable ${ }^{2}$}

7 La question du renforcement de la gouvernance internationale en matière environnementale et de développement durable, question récurrente, en particulier celle portant sur l'avenir du Programme des Nations Unies pour l'Environnement (PNUE) a été au centre des négociations. Ce cadre institutionnel du développement durable devait-il passer par un renforcement du PNUE ou par de nouvelles propositions innovantes, voire audacieuses, comme la création d'une Organisation Mondiale de l'Environnement (OME) sous la forme d'une véritable agence (option portée par la France, l'UE et les pays africains) ? Cette dernière option est restée au point mort et c'est en définitive (article 88) le renforcement du PNUE en tant qu'autorité mondiale reconnue en matière d'environnement qui a été retenu. Mais, en ce qui concerne la gouvernance mondiale du développement durable (article 84), l'Établissement d'un forum politique intergouvernemental de haut niveau pour remplacer la Commission de développement durable des Nations unies (CDD) marque une volonté de poursuivre les efforts. Également, concernant le renforcement du rôle de la société civile (article 84) au sein du forum politique intergouvernemental de haut niveau, il est prévu de renforcer le rôle consultatif et la participation des représentants de la société civile 
(accrédités auprès des Nations Unies) au niveau international afin de mieux faire usage de leur expertise, tout en conservant le caractère intergouvernemental des débats. Enfin, «Rio+20 » c'était encore une opportunité de s'adonner à une réflexion féconde sur les moyens de s'assurer que les trois piliers du développement durable font montre de la même solidité et de prendre des mesures hors du cadre des institutions dans lequel la communauté internationale fonctionne habituellement. L'enjeu ici était de traduire concrètement de façon non fragmentée le développement durable dans ses différentes composantes.

D'aucuns diront que le résultat est décevant, d'autres mettront plutôt l'accent sur la mobilisation de "tous", ceci étant entendu comme la société civile et les autorités locales, en dépit d'une rumeur persistante dénonçant l'adoption de la déclaration finale avant même l'ouverture du sommet. Si certains dénoncent « la dissipation de l'esprit de Rio » (Salmon, 2012), l'optimisme - fût-il mesuré - était de mise pour d'autres, mais tous considéraient qu'il aurait dû s'agir là d'un événement majeur sur la scène internationale, y compris ceux qui ont fait le choix de ne pas être à Rio et qui s'empressent de conclure sur : «Beaucoup de $\mathrm{CO}_{2}$ dépensé (ne serait-ce qu'en déplacement) pour finalement pas grand-chose?».

9 Faut-il attribuer ces critiques à des divergences entre acteurs sur la nature des thématiques abordées comme insatisfaisantes pour affronter les crises et dessiner des stratégies de développement soutenable ou faut-il considérer que la déclaration finale présente deux facettes, comme tout texte procédant d'une démarche de conciliation? Parfois, le compromis normatif ou conventionnel, fruit des négociations politiques s'avère, sinon nécessaire, pour le moins utile, dans l'évolution réelle de la prise en compte de la durabilité, même si elle se fait à petits pas, incomplète et loin de l'idéal. Ce que l'on peut, d'un côté, regretter en raison d'un texte procédant d'une affirmation insuffisante de la non-marchandisation de la nature peut, de l'autre, être perçu comme une opportunité de rénovation et d'obtention corrélative d'une plus grande effectivité, par la constitution d'un partenariat durable basé sur la gouvernance locale. En définitive, cette conférence n'était pas tant un événement commémoratif, qu'un nouveau souffle au développement durable. «Quel futur voulons-nous? », une question en suspens.

10 Si l'opportunité nous est donnée, dans le cadre de cet éditorial, d'évoquer des éléments d'actualité, ce numéro de développement durable et territoires se compose d'articles élaborés bien en amont de cette conférence. Le dossier "Trames vertes urbaines: recherches en sciences humaines et sociales » coordonné par Nathalie Blanc (CNRS, UMR Ladyss), Sandrine Glatron (CNRS, UMR LIVE) et Guillaume Schmitt (ULNF, UVHC) qui vous est proposé dans cette nouvelle livraison de Développement Durable et Territoires a pour objet d'étudier l'apport des recherches en sciences humaines et sociales sur les trames vertes urbaines. Les articles de ce dossier sont issus de l'ANR «ville durable (2008-2011)» et plus précisément d'un programme intitulé «Evaluation des Trame vertes urbaines et élaboration de référentiels : une infrastructure entre esthétique et écologie pour une nouvelle urbanité ". D'autres articles s'inscrivant dans ce même programme ANR TVU devraient être publiés dans la revue plus tard.

11 À travers des regards disciplinaires variés en SHS, le dossier explore la manière dont sont appréhendées des trames vertes concrètes ou projetées à la fois sous l'angle culturel, sociologique, géographique.

Bonne lecture! 


\section{BIBLIOGRAPHIE}

Aubertin C., 2012, « Repenser le développement du monde : le Brésil se met en scène à Rio +20 », Mouvement, $\mathrm{n}^{\circ} 70$, été, p. 43-58.

Gleizes J., 2012, « De 1992 à 2012, les sommets de la terre à Rio ou l'extension de la marchandisation du monde », Mouvement, n 70, été, p. 98-106.

Salmon J.M., 2012, « La dissipation de l'esprit de Rio », Mouvement, $\mathrm{n}^{\circ}$ 70, été, p. 14-30.

\section{NOTES}

1. L'économie verte est évoquée dans le point III de la déclaration finale : "L'économie verte dans le contexte du développement durable et de l'élimination de la pauvreté »; http:// www.uncsd2012.org/mgzerodraft.html, consulté le 30 juin 2012.

2. Ce thème est abordé au point IV de la déclaration finale: Dispositif institutionnel du développement durable ; http://www.uncsd2012.org/mgzerodraft.html, consulté le 30 juin 2012.

\section{AUTEUR}

\section{HELGA-JANE SCARWELL}

Helga-Jane Scarwell est professeure en Aménagement et Urbanisme à l'Université de Lille 1. Elle est membre du laboratoire TVES EA 4477. Ses travaux de recherche ont pour objet de souligner le caractère multiforme des enjeux environnementaux, à la fois sociaux, économiques, éthiques, politiques, industriels, scientifiques, technologiques, etc. helga.scarwell@univ-lille1.fr 